\title{
Nanomaterials for Organic Optoelectronic Devices: Organic Light-Emitting Diodes, Organics Solar Cells and Organic Gas Sensors
}

\author{
Nguyen Nang Dinh*, Tran Si Trong Khanh, Lam Minh Long, Nguyen Duc Cuong* and \\ Nguyen Phuong Hoai Nam
}

Faculty of Engineering Physics and Nanotechnology, VNU University of Engineering and Technology, Vietnam National University, Hanoi, 144 Xuan Thuy, Hanoi 10000, Vietnam

\begin{abstract}
This paper presents a unified view on applied nanomaterials that have been developed for a group of organic optoelectronic devices such as Organic Light-Emitting Diodes (OLEDs), Solar Cells (OSCs) and Gas Sensors (OGSs). From recent references, included our unpublished one, it has been demonstrated that nanostructured particles of metals, semiconductors, and oxides in conducting polymers embedded in conducting polymers have significantly contributed to improving both the performance parameters and working time of devices. The presence of inorganic nanoparticles in polymeric matrices has strongly influenced all physical properties of the polymers. However, herein the most interesting properties of OLEDs, OSCs, and OGSs are of electro-luminescence, photo-electrical conversion, and gas sensing, respectively. A publication has been seen regarding the nanostructured materials used for the fabrication of nanocomposite devices which aim at different practical purposes. [doi:10.2320/matertrans.MT-MN2019042]
\end{abstract}

(Received February 7, 2020; Accepted June 8, 2020; Published July 25, 2020)

Keywords: organic light-emitting diodes, organic solar cells, organic gas sensors, heterojunction, nanomaterials

\section{Introduction}

Over two recent decades, the interest of both theoretical and experimental investigation on organic materials included conducting polymers and organic devices has been fast increasing. This is because these materials exhibit numerous potential applications in organic electronics such as Organic Light-Emitting Diodes (OLEDs), Organic Solar Cells (OSCs), Organic Gas Sensors (OGSs), ${ }^{1-6)}$ etc. A significant advantage of conducting polymers above inorganic semiconductors is easy to adjust the energy band gap $\left(E_{\mathrm{g}}\right)$ between the highest occupied molecular orbital (HOMO) and the lowest unoccupied molecular orbital (LUMO) by combining conjugated polymers. Similar to inorganic semiconductors, when the polymers are excited by an energy $E \geq E_{\mathrm{g}}$, electrons from HOMO level jump to LUMO one (conducting band), at the same time holes appear in the HOMO (valence band). As a result, "electron-hole-pairs" (abbreviated to EHPs) or excitons are formed in polymers. In case an external electric field is applied to polymers, the electrons and the holes will move, respectively in LUMO and HOMO bands by opposite directions. By embedding nanostructured particles (shortly called "nanoparticles") of pure metals, semiconductors, and oxides in conducting polymers, one can improve both performance efficiency and service duration of the devices. There are many different techniques that have been used for producing nanomaterials and devices, with the aim to investigate the influence of the nanoparticles on the electrical and optical properties of conducting polymers. Among the optoelectronic applications of nanocomposites, herein we divide them into three areas that mostly concern to (i) process of the conversion from electrons to photons, namely electricity generates light (in OLEDs), (ii) process of the conversion from photons to electrons or light generates electricity (in solar cells) and

*Corresponding authors, E-mail: dinhnn@vnu.edu.vn, cuongnd@vnu.edu. $\mathrm{vn}$ (iii) the interaction of charges (included electrons and holes) with gases absorbed on the material surfaces. Most of the results summarized below are from the researches concerning nanoheterojunctions in conducting polymers. A part of the results reported in this review is taken from the published and unpublished of the authors and their collaborators in the University of Engineering and Technology, Vietnam National University, Hanoi (VNU-UET).

Abbreviations of main conducting polymers concerned in this paper are as follows:

$\begin{array}{ll}\text { MEH-PPV: } & \begin{array}{l}\text { Poly[2-methoxy-5-(2'-ethyl-hexyloxy)- } \\ \text { 1,4-phenylene vinylene] }\end{array} \\ \text { P3HT: } & \text { poly(3-hexylthiophene) } \\ \text { PFO: } & \text { Poly(9,9-di-n-octylfluorenyl-2,7-diyl) } \\ \text { PEDOT:PSS: } & \begin{array}{l}\text { Polyethylenedioxythiophene:poly(4-sty- } \\ \text { renesulfonate })\end{array} \\ \text { Py: } & \text { Pyrrole }\left(\mathrm{C}_{4} \mathrm{H}_{5} \mathrm{~N}\right)\end{array}$

\section{Organic Light-Emitting Diodes (OLED)}

OLEDs are different from LEDs in their structure, usually, an OLED is a multi-layers light-emitting diode containing at least one transparent electrically conducting film that is coated on glass flexible polyester foil substrates, and at least one thin flexible sheets of an organic electroluminescent material played a role of the emission layer (EML) that is positioned between two transparent substrates or between one transparent substrate and one mirror-like metallic layer. The light emitted from EML goes out through the transparent electrically conducting electrode (TCE) served as the anode. For OLEDs, the most TCE is a thin film of Sn-doped $\mathrm{In}_{2} \mathrm{O}_{3}$ (i.e., indium tin oxide, ITO). The ITO-glass substrate is coated with a hole transport layer (HTL), followed by the EML. The electron transport layer (ETL) is successively coated onto the EML, above which is a metallic (Al) cathode. The metallic cathode also plays a role of the mirror, reflecting light that is emitted from the EML. The top glass substrate is used for encapsulating devices. A DC-voltage applied 


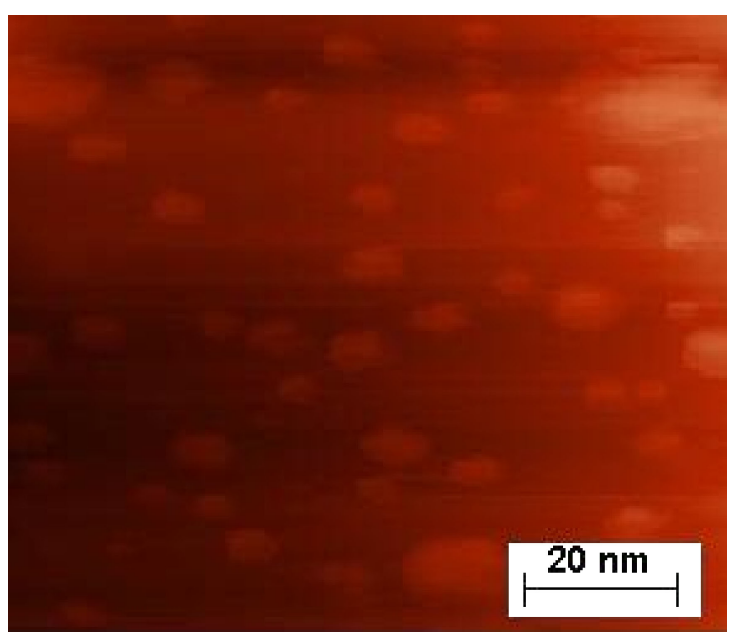

Fig. 1 AFM of a PEDOT-PSS $+\mathrm{TiO}_{2}$ composite film with the embedding of 20 wt. $\% \mathrm{TiO}_{2}$ nanoparticles. From Ref. 23).

between the ITO anode and Al cathode leads to creation of electron-hole-pairs, EHPs (i.e. excitons), then the recombination of EHPs results in emission of light. The value of this DC voltage is as low as $2-10 \mathrm{~V}^{7}$ )

For OLED devices, ITO has the most suitable working function, however, instead of ITO one can use $\mathrm{ZnO}: \mathrm{Al}$, $\mathrm{SnO}_{2}: \mathrm{F}^{8,9)}$ or a flexible anode like the single-walled carbon nanotubes (SWCNTs), ${ }^{10)}$ silver nanowires (AgNWs), ${ }^{11-13)}$ or graphene films ${ }^{14-16)}$ on flexible PET (polyethyleneterephthalate). In particular, Wang et al. ${ }^{10)}$ showed that, due to the room-temperature processing of SWCNTs films, the later can be prepared in large-area substrates for both top and bottom emission OLEDs. The EML, HTL and ETL thin films are different conducting polymers in almost all standard OLED devices. Recently, for improving efficiency and lifetime of devices, these layers were replaced by their composites with inorganic nanoparticles like $\mathrm{ZnO}, \mathrm{TiO}_{2}$, semiconducting QDs or carbon-based nanomaterials (CNT, rGO, graphene). ${ }^{17-20)}$

PEDOT-PSS is one of the best HTL used in OLED because it possesses not only high transmission in the visible region, good thermal endurance but also large conductivity. $^{21,22)}$ Nguyen et al. $^{23)}$ have shown that PEDOT-PSS/ITO interface contact can be improved by embedding $20 \mathrm{wt} . \%$ percentage of $\mathrm{TiO}_{2}$ nanoparticles in PEDOT-PSS (see Fig. 1). The high-resolution AFM picture has revealed a homogenous distribution of $\mathrm{TiO}_{2}$ nanoparticles in spin-coated PEDOTPSS. By FE-SEM micrographs, Nguyen et al. ${ }^{23)}$ also pointed out that in the composite sample the $\mathrm{TiO}_{2}$ nanoparticles with $5 \mathrm{~nm}$ in size are uniformly distributed. Due to the presence of the nanoparticles, both the roughness of the composite surface and PEDOT-PSS/ITO heterojunctions have been generated.

Using both the composite HTL and EML, Nguyen et al. ${ }^{23)}$ have prepared OLEDs. Firstly by spin-coating, the nanocomposite HTL film was deposited onto an ITO-coated glass substrate, followed by deposition nanocomposite EML (namely $\mathrm{MEH}-\mathrm{PPV}+\mathrm{nc}-\mathrm{TiO}_{2}$ ) onto the HTL. Then a shallow contact layer (SCL) of $\mathrm{Al} / \mathrm{LiF}$ was vacuum-evaporated onto the EML. Thus an OLED composite device with a laminar structure of $\mathrm{Al} / \mathrm{LiF} / \mathrm{MEH}-\mathrm{PPV}+\mathrm{nc}-\mathrm{TiO}_{2} / \mathrm{PEDOT}: \mathrm{PSS}+\mathrm{nc}-$ $\mathrm{TiO}_{2} /$ ITO was prepared. Characterization of the current-

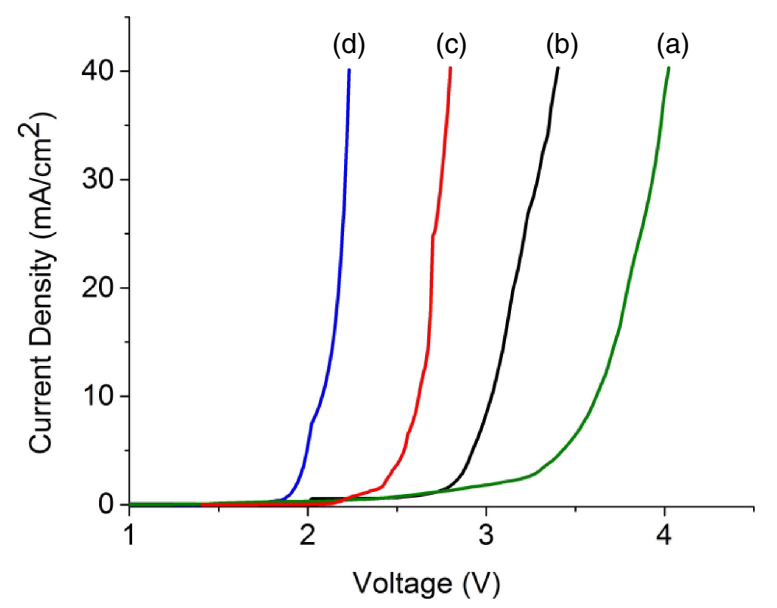

Fig. $2 I-V$ characteristics of OLED with different laminated structures. (a) - Single pristine MEH-PPV; (b) - with standard HTL layer; (c) - with HTL and EL composite layers and (d) - with SCL. From Ref. 23).

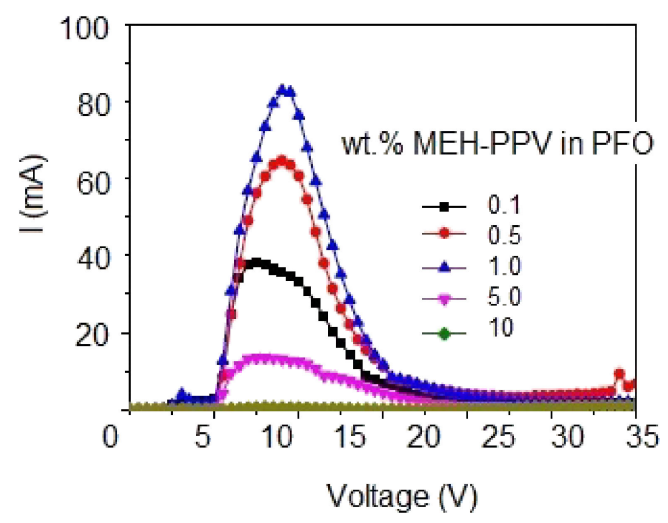

Fig. $3 \quad I-V$ characteristics of OLED based on PFO/MEH-PPV in the presence of $\mathrm{SiO}_{2} / \mathrm{TiO}_{2}$ nanocomposites. $1 \mathrm{wt} . \%$. is the optimal percentage of the MEH-PPV in PFO. From Ref. 24).

voltage ( $I-V$ curve) has demonstrated the significant enhancement in the electrical property of the nanocomposite OLED in comparison with that of the standard device (Fig. 2). The device with such an improved in $I-V$ characteristics has not only a larger efficiency but also better thermal stability due to eliminating the thermal power generated from Joule-Lenz current.

$\mathrm{Al}-\mathrm{Asbahi}^{24)}$ also reported the results on the OLEDs with both the $\mathrm{SiO}_{2} / \mathrm{TiO}_{2}+\mathrm{MEH}-\mathrm{PPV}$ and $\mathrm{SiO}_{2} / \mathrm{TiO}_{2}+\mathrm{PFO} /$ MEH-PPV (where PFO is Poly(9,9-di-n-octylfluorenyl-2,7diyl). They obtained a large improvement of the performance parameters of the composite OLED devices: The addition of $\mathrm{SiO}_{2} / \mathrm{TiO}_{2}$ in polymers enables the current to increase in more than 40-times in comparison with that of standard OLEDs, whereas the turn-on voltage decreased in more than three times (Fig. 3). Using Bis(3-(3,5-bis(dodecyloxy)phenyl)-(5-pyridin-2-yl)pyrazolate)platinum(II) complex (PT12) as a dopant in PFO, the color coordinates of these polymer OLEDs undergo a broad shift in the CIE color space. ${ }^{25)}$ Using this strategy, Cuerva et al. showed that white-OLED can be made with a suitable addition of PT12. Hybrid nanocomposites based on a mixture of Poly (9, 9-dioctyl-fluoenryl-2, 7-yleneethynylene) (PFE) and $\mathrm{ZnO}$ nanoparticles ( $n$ $\mathrm{ZnO})$ were prepared for OLED. $\left.{ }^{26}\right)$ For the PFE:ZnO based 
devices, the best performance parameters were obtained for 2 wt.\% of $\mathrm{ZnO}$ nanoparticles embedded in PFE.

In Ref. 20), Borriello et al. reported their work about OLED composite devices using $\mathrm{CdSe} / \mathrm{ZnS}$ quantum dots (QDs) combined with poly[(9,9-di-n-octylfluorenyl-2,7-diyl)alt-(benzo[2,1,3]thiadiazol-4,8-diyl)] (F8BT) for the EML. The last was prepared by two ways: to make a mixture of QDs with F8BT (forming so-called bulk heterojunctions) and to make a thin layer of QDs onto F8BT (planar heterojunction). The fluorescence of the mixture exhibit a broad emission peak from 500 to $700 \mathrm{~nm}$ due to the contribution of the emission from F8BT polymer (the peak at $540 \mathrm{~nm}$ ) and the emission from QDs (the peak at $634 \mathrm{~nm}$ ). Whereas for the planar structure, the QDs substantially contributes to the fluorescence spectrum. Herein, the authors of Ref. 20) have found out that all OLEDs made from EMLs with the mixture $15 \mathrm{wt} \%$ of QDs have the largest luminance efficiency. Although, their turn-on voltage is slightly changed comparing to pristine polymer devices.

Recently, Kaur et $a l .{ }^{27)}$ produced LED by using so-called Metal-Organic Frameworks (MOFs), because they have found that MOFs exhibited a good fluorescence lifetime, a large quantum efficiency and an excellent tunability. Embedding MOFs into OLEDs one can get enhanced values of CCT and CRI, consequently a wide range of the color emission spectrum. Therefore, the MOFs are expected to play important roles and have wide application in the OLED industry shortly.

\section{Organic Solar Cells}

Similar to OLEDs, OSCs usually have five layers, respectively positive electrode, HTL, photoactive layer, ETL and negative electrode. Positive electrode is a transparent electrically conducting film, negative electrode is usually a metallic or alloy thin film having a large reflectance. In the OLEDs, central layer is the EML (emitting layer), hereby in OSCs, it is the photoactive layer, consisting of $p-n$ junction of the donor and acceptor. A common architecture for highly-efficient OSCs is bulk heterojunction formed between fullerene derivatives (donor) and polymers (acceptor), as outlined in a recent book chapter. ${ }^{28)}$ Under the illumination of solar radiation, generated electrons jump from donor layer to acceptor one, leaving holes, creating the socalled EHPs (i.e. excitons). Due to a local electrical polarized voltage, the charge separation occurs: electrons move to the negative electrode and holes to the opposite one (positive electrode). In this way, an open voltage $\left(V_{\mathrm{oc}}\right)$ is created. When the circuit is short, one can have so-called short circuit current (in measurement it is usually taken in an area unit current density $-J_{\text {sc }}$ ).

Tran et al. ${ }^{30)}$ have demonstrated that using a nanocomposite photoactive layer, one can enhance the thermal endurance of the OSC devices. The thermal endurance and stability can be improved due to the lowering of the thermal expansion. As reported in Ref. 29), the thermal expansion coefficient $(\alpha)$ of a composite photoactive film (namely $\mathrm{P} 3 \mathrm{HT}+\mathrm{nc}-\mathrm{TiO}_{2}$ ) is nearly one order of magnitude smaller than that a pristine P3HT film. To compare the advantage of the composite-based OSCs when working at high temperatures, two types of OSCs have been made: one from pristine P3HT and the other from composite of P3HT and [6,6]phenyl C61 butyric acid methyl ester (PCBM). The thermal properties of both the photoactive materials and OSC devices have been characterized. AFM images shown in Fig. 4 have clearly revealed the annealing effect on the morphology of the two types of photoactive layers. In the polymer films annealed at $130^{\circ} \mathrm{C}$, the pores were largely expanded, but for the composite films annealed at the same temperature, no pores appeared. With the elimination of the pore in the photoactive layer, all the device performance parameters (namely $V_{\mathrm{oc}}, J_{\mathrm{sc}}$, and $\mathrm{FF}$ ) have been improved, consequently, the photoelectrical conversion efficiency (PCE) of the composite OSCs working at elevated operating-temperatures were found much better than the one of the standard devices.

A more detailed comparison between two types of the OSCs is shown in Ref. 30). The maximum PCE of the pristine $\mathrm{P} 3 \mathrm{HT}$ devices is ca. $1.6 \%$ (at a working temperature of $60^{\circ} \mathrm{C}$ ) and decreased to zero at $140^{\circ} \mathrm{C}$, whereas at this temperature the photoelectrical energy conversion process of the composite devices were still taken place and their PCE was maintained at a value as large as $1.2 \%$. This is attributed to the enhancement of the thermal property, namely thermal expansion coefficient $(\alpha)$ of the composite photoactive layer $\left(0.91 \times 10^{-5} \mathrm{~K}^{-1}\right)$ is much smaller than that of P3HT polymer $\left(7.60 \times 10^{-5} \mathrm{~K}^{-1}\right)$, as shown in Ref. 29). At a
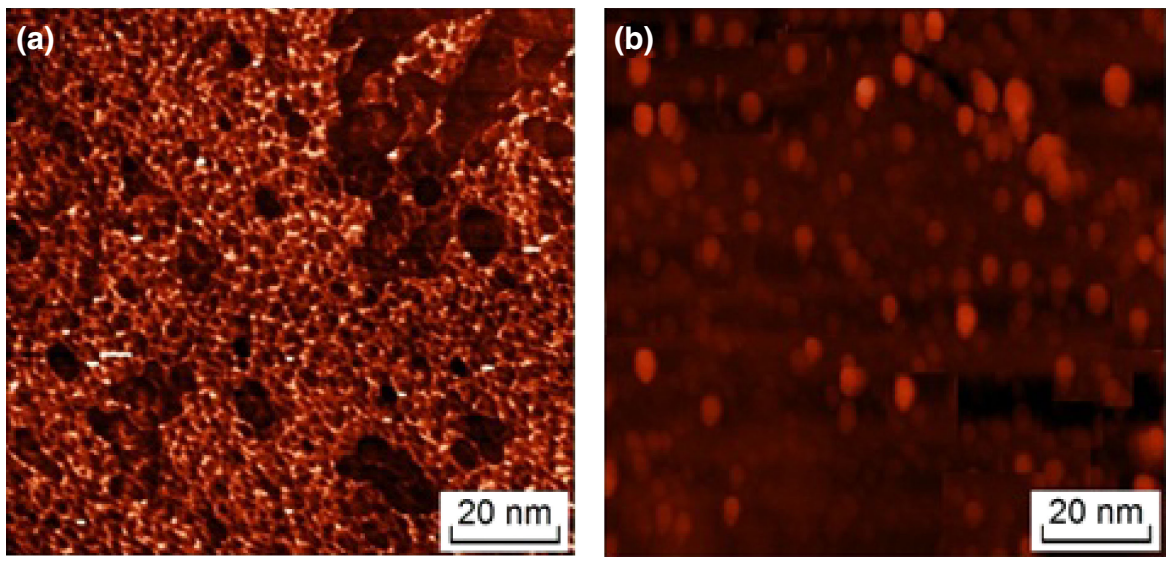

Fig. 4 AFM images of annealed films of P3HT (a) and $\mathrm{P} 3 \mathrm{HT}+5 \mathrm{wt} . \% \mathrm{TiO}_{2}$. Annealing temperature $T_{\mathrm{a}}=130^{\circ} \mathrm{C}$; the film thickness $d=100 \mathrm{~nm}$. From Ref. 30). 


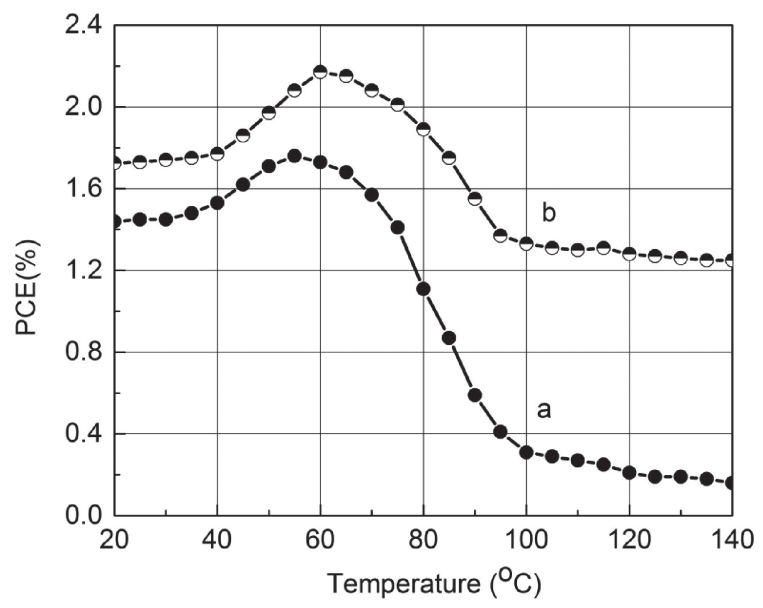

Fig. 5 Temperature dependence of the photoelectrical conversion efficiency of polymeric (curve "a") and composited-based devices (curve "b"). From Ref. 30).

high temperature, the appearance and fast growth of the pores in the polymer result in large defects that strongly trap charge carriers during their transport to both electrodes. On the contrary, in the composite photoactive layer, instead of the pores, there are numerous polymer $/ \mathrm{nc}-\mathrm{TiO}_{2}$ heterojunctions that facilitate charge carriers moving to the electrodes. Thus the PCE of the composite device is much improved comparing to those of the pristine-polymer device (Fig. 5). The fact that the PCE of composite OSCs attains large value at ca. $70^{\circ} \mathrm{C}$ showed that the effective temperature range $(60$ $80^{\circ} \mathrm{C}$ ) for polymeric photovoltaic devices is much lower than that for some types of inorganic solar cells $\left(100-170^{\circ} \mathrm{C}\right) .^{31)}$

Metal-oxide nanoparticles (NPs) have also been used in both organic solar cells and hybrid organic-inorganic perovskite solar cells (PSCs), as summarized in a recent review paper. ${ }^{32)} \mathrm{ZnO}, \mathrm{TiO}_{2}$ NPs serve as electron-transport materials, ${ }^{33,34)}$ while $\mathrm{NiO}_{\mathrm{x}}$, Cu-doped $\mathrm{NiO}_{\mathrm{x}} \mathrm{NPs}$, and graphene oxide functionalized with $\mathrm{MoO}_{\mathrm{x}} \mathrm{NPs}$ serve as hole-transporting materials. ${ }^{35-37)}$ In addition to the wide bandgap (i.e. high transmittance), another important advantage of metal oxide NPs is that the technology for the films prepared by solution-process and at low temperatures has a reduced cost, hence they are suitable to be used in solar cells with conventional and inverted structures. ${ }^{38,39)}$ The low annealing temperature (typically $<100^{\circ} \mathrm{C}$ ) required to prepare films from metal oxide nanoparticles also allows coating directly on flexible substrates, as well as on ITO substrates without increasing their sheet resistance. ${ }^{40)}$ Moreover, metal oxide NPs layers could efficiently enhance the stability of OSCs and PSCs in the open air, especially when they are formed on top of the active layers. ${ }^{41,42}$ ) The combinations of active layers with nanostructured metal oxides like $\mathrm{ZnO}$ nanorods (NRs) or $\mathrm{TiO}_{2}$ NRs were also investigated. ${ }^{43-48)}$ The aim of utilizing this combination is to enhance charge separation efficiency. Nguyen et al. ${ }^{49)}$ have demonstrated that with the addition of a thin $\mathrm{ZnO}$ film (30$\mathrm{nm})$ served as a buffer layer between the ITO electrode and the hole transport layer, the fill factor of the OSCs considerably enhanced, consequently, the PCE increased about $20 \%$, namely from PCE $=1.75 \%$ (for devices without $\mathrm{ZnO}$ ) to $2.12 \%$ (for devices with $\mathrm{ZnO}$ ).
The localized surface plasmonic resonance (LSPR) has strongly affected to $\eta$ of OSCs, ${ }^{50)}$ this is due to the exciton dissociation enhancement, improved charge transport assisted with reduced recombination, and the crystallinity enhancement of the polymer/fullerene bulk-heterojunctions (BHJ) blend for enhanced charge transport and collection. ${ }^{51)}$ The LSPR effect of metal nanoparticles (NPs) enhances the electromagnetic field which expedites photoabsorption and generation of excess exciton in the photoactive layer of a photovoltaic device while the light scattering effect increases the optical path length within the active layer which is also beneficial to exciton generation. ${ }^{52)}$ Moreover, exciton diffusion and charge dissociation are also encouraged when the electromagnetic field of the metal NPs interact with the generated excitons. However, despite being applicable in all functional layers of BHJ OSC, ${ }^{53)}$ metal NPs are more preferable when they are rightly incorporated in the photoactive layer because the LSPR effect is restricted to a fewnanometer scale. ${ }^{54)}$ For the best performance enhancement in BHJ SC, the position of nanoparticles in the device's structure also depends on the particle size. ${ }^{55)}$ Various reports involving the incorporation of nanocomposites in the photoactive layer have been demonstrated widely by researchers to improve all the performance parameters of the photovoltaic devices. For hybrid perovskite solar cells (PSCs), Luo et al. indicated that by embedding $\mathrm{Au} @ \mathrm{TiO}_{2}$ NPs into both the porous $\mathrm{TiO}_{2}$ and perovskite capping layers, the PCE of the PSCs has increased from $12.59 \%$ to $18.24 \% .{ }^{56)}$ The increase in the PCE is explained due to the enhancement of the $J_{\mathrm{sc}}$, the last is consistent with the EQE enhancement over the visible region (from $400 \mathrm{~nm}$ to $750 \mathrm{~nm}$ ).

\section{Organic Film Gas Sensors}

Resistive organic film gas sensor (OGS) usually has a structure, which is much simpler comparing to those of both OLED and OSC. The OGS device has one polymeric composite layer coated on a transparent substrate (corning glass), two Pt electrodes in a few $\mu \mathrm{m}$ separated each from other. The organic nanocomposite film consists of a polymer matrix (PEDOT:PSS or P3HT, PFO, etc.) and inorganic nanoparticles (NP) like $\mathrm{TiO}_{2}, \mathrm{ZnO}, \mathrm{CNTs}$, GQDs. When gas is absorbed on the film surface, the resistance of the film changes as a function of gas content. Such a sensor is called a resistive gas sensor. From its function, one can monitor the concentration of gases as small as a few ppm. ${ }^{57)} \mathrm{A}$ voltammetric sensor based on a polyaniline/graphene oxide modified glassy carbon electrode (PANI/GRO/GCE) was prepared for the ultrasensitive voltammetric detection of clonazepam (CZPM). ${ }^{58)}$ Due to the synthetic PANI/GRO nanocomposite along with its precursors PANI and GRO, the catalytic response of the PANI/GRO/GCE was enhanced with a reproducibility of $4.21 \%$ relative standard deviation (RSD) and a satisfactory recovery from $97.16 \%$ to $102.78 \%$.

Olenych et al. ${ }^{59)}$ showed that for composite films made from PEDOT:PSS with porous silicon (PS) and CNTs, the considerable influence of atmospheric conditions like relative humidity to both the electrical resistance and capacitance of the composite films. The dynamic dependencies for the PEDOT:PSS-PS-CNT composite sensor were characterized 
by $R$ - $t$ and $C-t$ curves. In an earlier work, ${ }^{60)}$ these dependencies were attributed to the interaction of water molecules with the composite surface, resulting in the increase of electrical conductivity of both the PS nanocrystals and hybrid films. Ouyang et al. ${ }^{21)}$ explained the change in the conductivity of the conjugated polymer (PEDOT:PSS) due to the water molecules doped in the polymers.

The performance of $\mathrm{NH}_{3}$ sensor is similar to the moisture sensor. Wang et al. ${ }^{61)}$ carried-out the detailed characterization of $\mathrm{NH}_{3}$ gas sensors made from reduced graphene oxide (rGO)-based films. They showed that the resistance of sensors much increased with the increase of $\mathrm{NH}_{3}$ gas concentration. Moreover, the different response to $\mathrm{NH}_{3}$ gas was obtained for pyrrole-vapor-reduced rGO (Py-rGO) and hydrazine-vapor-reduced rGO (Hy-rGO) sensors: The first device expressed a much better response to $\mathrm{NH}_{3}$ in comparison to the second one. The largest response of the first sensor was achieved when a $0.5 \mathrm{mg} / \mathrm{mL}$ concentration of rGO was added in Py-rGO, while for the second sensor, it was of $0.25 \mathrm{mg} / \mathrm{mL}$ (Fig. 6). When exposing to $\mathrm{NH}_{3}$ gas with $50 \mathrm{ppm}$ concentration, the response of Py-rGO sensors increased in more than 2.7 times, moreover, better responsive repeatability to $\mathrm{NH}_{3}$ of the devices has also been obtained. The variations of the sensitivity of sensor devices by the change of the assembly concentration of $\mathrm{rGO}$ were clarified by Wang et al. ${ }^{61)}$ With the increase of rGO-solution concentration, the response of Hy-rGO-based sensors decreased from $5.3 \%$ to $1.6 \%$. The authors in Ref. 61) have explained this dependence due to following facts: (i) As the large size of graphene sheets appeared, fewer ammonia molecules diffused inside the composite film; (ii) More graphene sheets are stacked each onto other, forming socalled dense rGO structure and thus the porosity of the film is much lowering. These facts have made the $\mathrm{NH}_{3}$ adsorption by the film much more difficult. Patil et al. ${ }^{62)}$ have also observed a similar effect when using Py-rGO sensors for monitoring both the $\mathrm{H}_{2} \mathrm{~S}, \mathrm{LPG}, \mathrm{CO}_{2}$ and $\mathrm{NH}_{3}$ gases. Dunst et al. ${ }^{63)}$ have characterized the sensing properties of GO, rGO, and PEDOT-rGO films towards relative humidity and different gases, and have shown that the best response was observed for $\mathrm{NO}_{2}$ gas.

When the commercial CNTs embedded in polymers, one must treat their surface modification for better polymer/CNT heterojunction interface. However, by the modification, some uncontrolled impure compounds usually appear and diffuse inside devices, causing charge traps or destroying of their composition. Recently, to avoid this effect, we have used directly pitch to make the so-called carbon replica (CR). Pitch, as well known, is a carbon-rich material, thus it can be used for carbon-contained precursors. From the last one can make a well-defined and ordered graphene structure. ${ }^{64)}$ Using a nano-casting approach, Birte Jache et al. ${ }^{65)}$ have prepared carbon materials with defined porosity. Where the $\mathrm{SiO}_{2}$ template used for experiments has a few hundred nanometers. This value is considerably large for making nanoheterojunctions. Thus, we followed the nano-casting method described in Ref. 65) but using the silica template of ca. $10 \mathrm{~nm}$ in size. The pitch used for this was brought from Thai-Nguyen (Vietnam) coal tar. Mesophase pitch (MP) of the coal tar was dissolved in tetrahydrofuran (THF) solution containing $10 \mathrm{wt} \%$ of MP (MPT) using ultrasonic stirring for $3 \div 5 \mathrm{~h}$. Then using centrispining technique with $v=4,000 \mathrm{rpm}, t=$ $5 \mathrm{~min}$, the MPT solution was poured off. In our process, $100 \mathrm{mg}$ of silica nanopowder was completely dispersed in the MPT solution (abbreviated to MPS). Using magnetic stirring $\left(T=45^{\circ} \mathrm{C}, t=90 \mathrm{~min}\right)$, silica nanoparticles were covered by a carbon-rich thin layer. The solvent of the MPS solution then was taken out by continually ultrasonic-stirring at $45^{\circ} \mathrm{C}$ until the solvent was completely removed. The obtained product was a nanocomposite of pitch- $\mathrm{SiO}_{2}$, where the spherical nano- $\mathrm{SiO}_{2}$ was coated by a carbon-rich thin layer. The pores between $\mathrm{SiO}_{2}$ nanoparticles were also filled. Under a dry Ar ambiance in a vacuum chamber, the pitch$\mathrm{SiO}_{2}$ nanocomposite was carbonized at a temperature of $450^{\circ} \mathrm{C}$, for $2 \mathrm{~h}$. This carbonized pitch- $\mathrm{SiO}_{2}$ product was put in a $\mathrm{HF}$ solution. In this way, the $\mathrm{SiO}_{2}$ templates were removed, forming the spherical CR nanomaterial. Since the silica nanoparticles were closely contacted each to other, the $\mathrm{CR}$ pores were well connected. Then using the $\mathrm{CR}$, we prepared P3HT-CR (abbreviated to P3C) composite films for $\mathrm{NH}_{3}$ gas sensing.

For this, firstly $\mathrm{P} 3 \mathrm{HT}$ powder $(8 \mathrm{mg}$ ) was completely dissolved in chlorobenzene $(1 \mathrm{ml})$ due to ultrasonic stirring for $15 \mathrm{~min}$. For preparing the $\mathrm{P} 3 \mathrm{C}$ composites, above mentioned $\mathrm{CR}$ were added in the P3HT solution with a weight ratio of $\mathrm{CR} /$ polymer equal to 0.10 (namely $10 \mathrm{wt} . \%$ ).
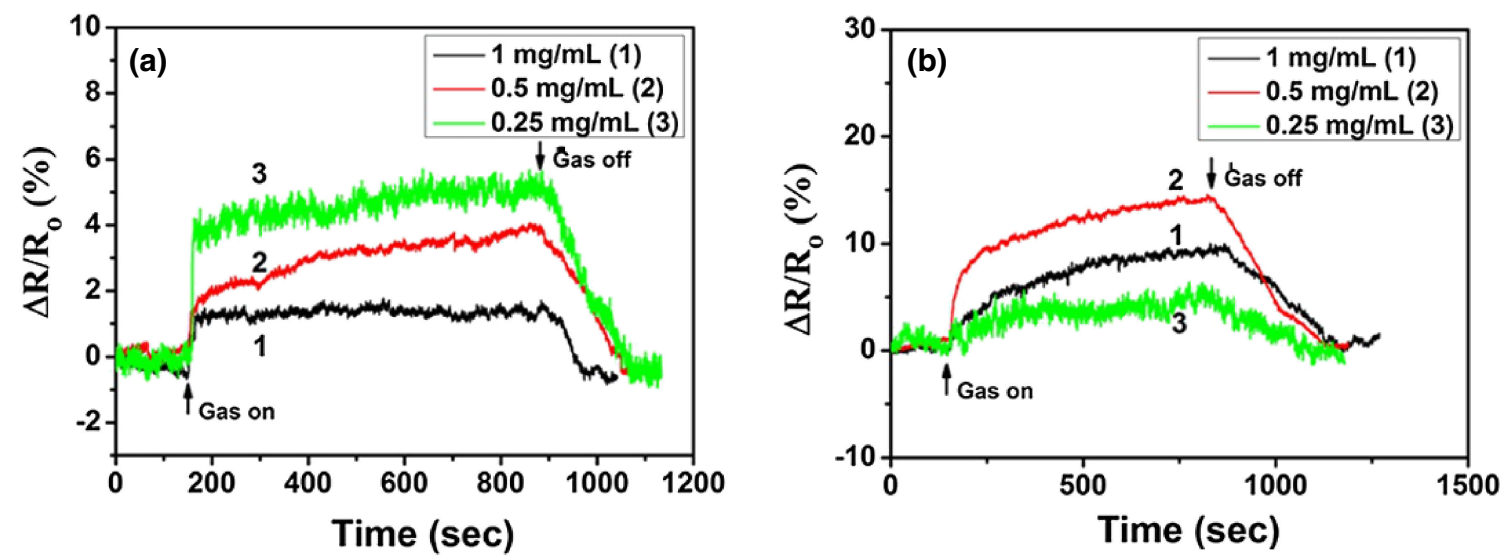

Fig. 6 Plot of normalized resistance change versus time for the sensing devices based on Hy-rGO (a) and Py-rGO (b) fabricated with GO assembly concentration at $1,0.5$, and $0.25 \mathrm{mg} / \mathrm{mL}$ (the concentration of $\mathrm{NH}_{3}$ gas is $50 \mathrm{ppm}$ ). From Ref. 61). 


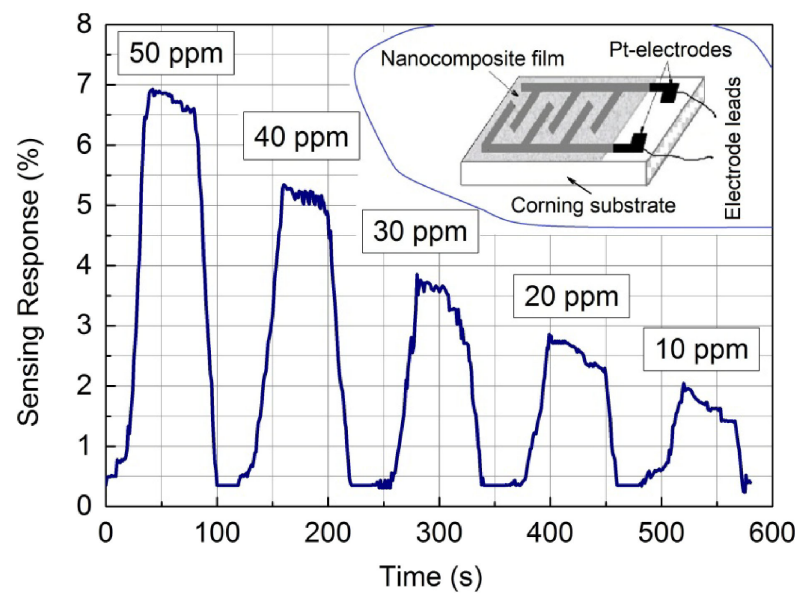

Fig. 7 Time dependence of the sensing response of $\mathrm{P} 3 \mathrm{C}$ film on repeated exposure and removal of $\mathrm{NH}_{3}$ gas (unpublished results). Insertion is the schematic drawing of the $\mathrm{P} 3 \mathrm{C}$ sensor.

To get a well-dispersed nano-CR in the polymer, the P3C solution was stirred for $8 \mathrm{~h}$ by using a stirring bar. Further, this $\mathrm{P} 3 \mathrm{C}$ solution was solidified for gas-sensors preparation. The $70 \mathrm{~nm}$-thickness Pt-coated glass was used for two electrodes. The procedure for spin-coating was repeated as reported in Ref. 30): The technical parameters such as delay time, rest time, spin speed, acceleration, and drying time were chosen as $120 \mathrm{~s}, 30 \mathrm{~s}, 1800 \mathrm{rpm}, 500 \mathrm{rpm}$, and $180 \mathrm{~s}$, respectively. $\mathrm{P} 3 \mathrm{C}$-composite sensor has a structure shown in the insertion of Fig. 7. The thickness of the P3C composite films for $\mathrm{NH}_{3}$ sensing is about $400 \mathrm{~nm}$. For sensing characterization toward each device, we have taken a number of the measurements as large as 10 cycles. The $\mathrm{NH}_{3}$ gas flow introduced in the measuring chamber during each test was $60 \mathrm{sccm}$ (or $\mathrm{ml} / \mathrm{min}$ ). In the P3HT-CR composite film, together with numerous nano-heterojunctions of the P3HT/ carbon sphere, the charge traps have been almost eliminated. Without the charge traps, one can expect a considerable enhancement of performance parameters of the composite sensors like response time, sensing response, etc. The sensing response $(\delta)$, as well-known, is characterized by the following formula:

$$
\delta=\frac{R-R_{o}}{R_{0}}(\%)
$$

where $R_{\mathrm{O}}$ is the value of "ex situ" resistance (i.e. initial resistance) of the sensors; $R$ - the "in situ" resistance of the sensor, namely the resistance obtained under the exposure in the monitoring gas. The concentration $\left(C_{\mathrm{gas}}\right)$ of $\mathrm{NH}_{3}$ gas introduced into the measuring chamber was chosen with decreasing from $50 \mathrm{ppm}$ to $40,30,20$ and $10 \mathrm{ppm}$. To findout the limitation of the gas detecting, the $\mathrm{P} 3 \mathrm{C}$ composite sensors were exposed to $\mathrm{NH}_{3}$ gas, in turn of $C_{\text {gas }}$ ranging from $50 \mathrm{ppm}$ to $10 \mathrm{ppm}$. By measuring the sensor resistance versus the concentration of $\mathrm{NH}_{3}$ gas in/out measuring chamber as well as measurement time, one can characterize the repeatability of the resistance change of the composite. A measurement cycle consists of the time of $\mathrm{NH}_{3}$ gas exposure and its extraction out from the chamber. In our experiments, $30 \mathrm{~s}$ was chosen for $\mathrm{NH}_{3}$ exposure in the chamber with the above-mentioned gas concentrations (namely 50, 40, 30, 20 and $10 \mathrm{ppm}$ ), and 70 seconds - for extraction of ammonia gas out. An additional time of $20 \mathrm{~s}$ was taken for heating the sensor in order the desorption of the $\mathrm{NH}_{3}$ gas was completed, consequently, the prior cycle was also ended. The next measurement cycle was carried-out in the same procedure.

Figure 7 demonstrates the experimental results obtained in 5 cycles of the measurements for the sensing response, respectively for $\mathrm{NH}_{3}$ concentration decreasing from 50 to 40 , 30, 20, and $10 \mathrm{ppm}$. From Fig. 7, one can see that the P3C composite sensors exhibited a responding time as low as $30 \mathrm{~s}$. Additionally, the resistance of the sensor recovered to its initial value when exposed to a standard atmosphere. In the same period of the $\mathrm{NH}_{3}$ gas exposure (namely $30 \mathrm{~s}$ ), the sensing response to $\mathrm{NH}_{3}$ gas concentration ranging from 50 to $40,30,20$, and $10 \mathrm{ppm}$ was found to be of 2.9 , to $2.4,1.8$, 1.3 and $0.8 \%$, respectively. This means that the sensing response of the sensors decreases with the decrease of the concentration of the exposure $\mathrm{NH}_{3}$ gas. From Fig. 7 it is seen that the sensing response can be well revealed when the $\mathrm{NH}_{3}$ concentration is less than $10 \mathrm{ppm}$. Thus an advantage of the sensors made from P3HT-CR composite is the enhancement of the detection limit towards $\mathrm{NH}_{3}$ gas monitoring. In our present work, this detection limit is not evident, since the EPA-MTH gas profilometer used in experiments is impossible to control a $\mathrm{NH}_{3}$ gas flow with an accurate concentration less than $10 \mathrm{ppm}$.

In order to improve the sensitivity of $\mathrm{NH}_{3}$ gas sensor, some noble metals (Pt, Ag) were used to decorate CNTs network. ${ }^{66)}$ In another report, $\mathrm{Ni}_{2} \mathrm{O}_{3}$ nanoparticles were utilized to decorate $\mathrm{WO}_{3}$ film for high sensitivity $\mathrm{NH}_{3}$ gas sensor. ${ }^{67}$ )

\section{Discussion}

In almost organic optoelectronic and electronic devices, the inorganic nanoparticles embedded in polymeric matrices enable devices to enhance their performance parameters, including an important one - the efficiency of the devices.

Firstly, for OLEDs, it was observed that the embedding inorganic nanoparticles (f.i. $\mathrm{TiO}_{2}$ ) in conducting polymers have filled up pinholes/nano-pores as so-called charge traps that appear in polymers due to the thermal annealing. The centrispinning force in the spin-coating technique enables the nanoparticles to adhere stronger to both HTL and EML layers. Thus the $I-V$ curve of the device is improved (see "c" curve in Fig. 2). The addition of the LiF nanolayer served as shallow contact between EML and Al-cathode has made more efficient electron injection from the cathode into EML, resulting in the steepest $I-V$ characteristic (see "d" curve in Fig. 2). Tsutsui et al. ${ }^{68)}$ have shown that the electroluminescence quantum efficiency can be calculated by following the equation:

$$
\eta=\gamma \times \eta_{r} \times \phi_{f}
$$

where $\gamma$ is a factor expressing the injection of electrons and holes. Its maximal value is equal to 1 only when the balanced charge injection into the EML of the device occurs. In the other words, the numbers of injected electrons and holes are the same; $\eta_{r}$ is a factor determining the efficiency of the singlet-exciton formation from a positive and a negative polaron, and $\phi_{f}$ is the photoluminescence quantum efficiency. 
The enhancement in both the photoluminescence spectra and the current-voltage characteristics as shown by Nguyen et $a .^{23)}$ demonstrates that the factor $\gamma$ of the devices made from both the composite HTL and EML attained the largest value. Due to the $\mathrm{TiO}_{2} /$ polymer junctions, more mobile electrons from cathode faster move to EML where holes have injected to. Consequently, a larger number of EHPs is formed, as a result, the factor $\eta_{r}$ increases. Thus the electroluminescence quantum efficiency of composite-based OLEDs is much larger than the one of the standard polymeric devices. From our experiments, it has been seen that $\eta$ of the composite devices increased in an order of magnitude in comparison with the standard devices. ${ }^{23)}$

Secondly, for OSCs, Tran et al. ${ }^{30)}$ showed that in photoactive polymeric layers annealed at a temperature range of 70 to $140^{\circ} \mathrm{C}$ or during the expose under elevated operating-temperatures a large number of nanopores/cracks occurred. They are considered as defects, strongly trapping both electrons and holes. But in the polymeric composite film, the nanoparticles have filled up these defects, replacing by numerous nanoparticles/polymer heterojunctions. Thus the generated electrons in the photoactive layers move faster to an electrode, while the holes move to the other one. As a result, the stronger charge-separation effect occurs in the composite devices, hence the performance parameters of composite devices (namely $V_{\mathrm{oc}}, J_{\mathrm{sc}}$, and FF) have much improved. Thus the photoelectrical conversion efficiency of the composite OSCs increases.

Finally, for OGS, embedding nanoparticles included carbon spheres in polymers (in our experiments) has also resulted in a significant enhancement of the sensing performance of many organic gas sensors. Herein, there is a brief review on $\mathrm{NH}_{3}$ gas sensors. Ammoniac molecule $\mathrm{NH}_{3}$, as a reducing agent, has a lone electron pair that can be easily donated to $p$-type conducting polymers (f.i. P3HT), resulting in lowering hole concentration. Therefore the resistance of the sensors decreases. In CRs-P3HT composite films, there are numerous polymeric nanospheres covered by thin carbon layers (TCL). These TCLs have contributed to improving the adsorption efficiency of gas-molecules due to larger effective surface areas. Moreover, the addition of TCLs in P3HC films has created nanostructured heterojunctions, strongly favoring charge transferring. Consequently, the sensing response of the resistive sensors is improved. It is worthwhile noting that in this review, we discussed mainly the combination of organic compounds with carbon-based ones. The readers are encouraged to find a detailed review, in which gas sensors using heterojunction between graphene and its derivatives with other inorganic compounds have been discussed. ${ }^{69}$

\section{Summary}

This topical brief review delineates a set of nanotechnologies and characterization for electronic and optoelectronic devices such as OLEDs, OSCs, and Organic Gas Sensors that may effectively contribute further to organic electronics. The advantage of nanoparticles embedded in conducting polymers has been elucidated, basing on the enhancement of performance parameters of all the composite-based devices. Taking an appropriate choice of the combination between the conducting polymers and inorganic nanoparticles, it is possible (i) to eliminate nanopores and/or cracks in polymers, and (ii) to make numerous heterojunctions in the composites that enable the devices to improve their performance parameters and service duration, when the devices work at elevated operatingtemperatures, in particular.

\section{Acknowledgements}

This research is funded by Vietnam National Foundation for Science and Technology Development (NAFOSTED) under grant number 103.02-2018.55.

\section{REFERENCES}

1) M. Zhang, S. Höfle, J. Czolk, A. Mertens and A. Colsmann: Nanoscale 7 (2015) 20009-20014.

2) D. Luo, Q. Chen, B. Liu and Y. Qiu: Polymers (Basel) 11 (2019) 384

3) G. Li, R. Zhu and Y. Yang: Nat. Photonics 6 (2012) 153-161.

4) Y. Gao, Z. Wang, G. Yue, X. Yu, X. Liu, G. Yang, F. Tan, Z. Wei and W. Zhang: Sol. RRL 3 (2019) 1900012.

5) Z. Zhang, J. Miao, Z. Ding, B. Kan, B. Lin, X. Wan, W. Ma, Y. Chen, X. Long, C. Dou, J. Zhang, J. Liu and L. Wang: Nat. Commun. 10 (2019) 3271

6) N.L. Torad and M.M. Ayad: Gas Sensors, (IntechOpen, 2019).

7) R. Mertens (ed.): The OLED Handbook, (Lulu, Raleigh, 2019) Paperback, p. 200.

8) Y.J. Choi, S.C. Gong, C.S. Park, H.S. Lee, J.G. Jang, H.J. Chang, G.Y. Yeom and H.H. Park: ACS Appl. Mater. Interfaces 5 (2013) 36503655 .

9) D. Saikia and R. Sarma: J. Electron. Mater. 47 (2018) 737-743.

10) K. Wang, H. Dong, D. Zhou, Y. Ito, L. Hu, Z. Zhang and X. Zhu: ACS Appl. Mater. Interfaces 12 (2020) 8722-8729.

11) W. Li, A. Meredov and A. Shamim: Npj Flex. Electron. 3 (2019) 19.

12) S. Coskun, E.S. Ates and H.E. Unalan: Nanotechnology 24 (2013) 125202.

13) Q. Nian, M. Saei, Y. Xu, G. Sabyasachi, B. Deng, Y.P. Chen and G.J. Cheng: ACS Nano 9 (2015) 10018-10031.

14) T.H. Han, Y. Lee, M.R. Choi, S.H. Woo, S.H. Bae, B.H. Hong, J.H. Ahn and T.W. Lee: Nat. Photonics 6 (2012) 105-110.

15) J.T. Lim, H. Lee, H. Cho, B.H. Kwon, N.S. Cho, B.K. Lee, J. Park, J. Kim, J.H. Han, J.H. Yang, B.G. Yu, C.S. Hwang, S.C. Lim and J. Lee: Sci. Rep. 5 (2015) 17748.

16) S. Haque, M.S.A. Sher Shah, M. Rahman and M. Mohiuddin: Biopolym. Compos. Electron., (Elsevier Inc., Amsterdam, 2017) pp. 277-310.

17) D. Dong, Y. Wang, L. Lian, D. Feng, H. Wang and G. He: Phys. Status Solidi A 214 (2017) 1700583.

18) V. Mann and V. Rastogi: Opt. Commun. 387 (2017) 202-207.

19) C.Y. Park and B. Choi: Nanomaterials 9 (2019) 1241.

20) C. Borriello, C.T. Prontera, S.A. Mansour, S. Aprano, M.G. Maglione, A. Bruno, T.D. Luccio and C. Minarini: Phys. Status Solidi C 12 (2015) 1416-1420.

21) J. Ouyang, Q. Xu, C.-W. Chu, Y. Yang, G. Li and J. Shinar: Polymer 45 (2004) 8443-8450

22) P. Tehrani, A. Kanciurzewska, X. Crispin, N.D. Robinson, M. Fahlman and M. Berggren: Solid State Ionics 177 (2007) 3521-3527.

23) N.D. Nguyen, H.C. Le, T.C.T. Tran, Q.T. Tran and V.T. Vo: J. Appl. Phys. 105 (2009) 093518.

24) B.A. Al-Asbahi: Polymers (Basel) 10 (2018) 800.

25) C. Cuerva, J.A. Campo, M. Cano, B. Arredondo, B. Romero, E. Otón and J.M. Otón: New J. Chem. 39 (2015) 8467-8473.

26) M. Belhaj, C. Dridi and H. Elhouichet: J. Lumin. 157 (2015) 53-57.

27) H. Kaur, S. Sundriyal, V. Pachauri, S. Ingebrandt, K.H. Kim, A.L. Sharma and A. Deep: Coord. Chem. Rev. 401 (2019) 213077.

28) I.E. Kuznetsov, A.V. Akkuratov and P.A. Troshin: Nanomater. Sol. Cell Appl., (Elsevier, Amsterdam, 2019) pp. 557-600.

29) V.T. Nguyen, V.S. Tran, Q.T. Tran, T.T. Tran and N.D. Nguyen: VNU J. 
Sci.: Math. Phys. 31 (2015) 21-27.

30) T.T. Tran, Q.T. Tran, V.T. Vo and N.D. Nguyen: J. Nanomater. 2015 (2015) 463565.

31) D. Meneses-Rodríguez, P.P. Horley, J. González-Hernández, Y.V. Vorobiev and P.N. Gorley: Sol. Energy 78 (2005) 243-250.

32) D. Ouyang, Z. Huang and W.C.H. Choy: Adv. Funct. Mater. 29 (2019) 1804660 (Review).

33) Z. Zheng, S. Zhang, J. Wang, J. Zhang, D. Zhang, Y. Zhang, Z. Wei, Z Tang, J. Hou and H. Zhou: J. Mater. Chem. A 7 (2019) 3570-3576.

34) H. Zhou, Q. Chen, G. Li, S. Luo, T.-B. Song, H.-S. Duan, Z. Hong, J. You, Y. Liu and Y. Yang: Science 345 (2014) 542-546.

35) U. Kwon, B.-G. Kim, D.C. Nguyen, J.-H. Park, N.Y. Ha, S.-J. Kim, S.H. Ko, S. Lee, D. Lee and H.J. Park: Sci. Rep. 6 (2016) 30759.

36) S. Huang, Y. Wang, S. Shen, Y. Tang, A. Yu, B. Kang, S.R.P. Silva and G. Lu: J. Colloid Interface Sci. 535 (2019) 308-317.

37) S.S. Bhosale, E. Jokar, A. Fathi, C.M. Tsai, C.Y. Wang and E.W. Guang Diau: Adv. Funct. Mater. 28 (2018) 1803200.

38) S.H. Oh, S.J. Heo, J.S. Yang and H.J. Kim: ACS Appl. Mater. Interfaces 5 (2013) 11530-11534.

39) J. You, C.C. Chen, L. Dou, S. Murase, H.S. Duan, S.A. Hawks, T. Xu, H.J. Son, L. Yu, G. Li and Y. Yang: Adv. Mater. 24 (2012) 5267-5272.

40) S.K. Hau, H.L. Yip, N.S. Baek, J. Zou, K. O’Malley and A.K.Y. Jen: Appl. Phys. Lett. 92 (2008) 253301.

41) J. You, L. Meng, T.B. Song, T.F. Guo, W.H. Chang, Z. Hong, H. Chen, H. Zhou, Q. Chen, Y. Liu, N.D. Marco and Y. Yang: Nat. Nanotechnol 11 (2016) 75-81.

42) H. Zhang, J. Cheng, F. Lin, H. He, J. Mao, K.S. Wong, A.K.-Y. Jen and W.C.H. Choy: ACS Nano 10 (2016) 1503-1511.

43) L. Feng and X.T. Hao: Mater. Trans. 58 (2017) 1106-1110.

44) N.C. Das, S. Biswas and P.E. Sokol: J. Renew. Sustain. Energy 3 (2011) 033105.

45) P. Ruankham, L. Macaraig, T. Sagawa, H. Nakazumi and S Yoshikawa: J. Phys. Chem. C 115 (2011) 23809-23816.

46) A. Loiudice, A. Rizzo, L.D. Marco, M.R. Belviso, G. Caputo, P.D. Cozzoli and G. Gigli: Phys. Chem. Chem. Phys. 14 (2012) 3987-3995.

47) D.Y. Son, J.H. Im, H.S. Kim and N.G. Park: J. Phys. Chem. C 118 (2014) 16567-16573

48) X. Li, S.M. Dai, P. Zhu, L.L. Deng, S.Y. Xie, Q. Cui, H. Chen, N. Wang and H. Lin: ACS Appl. Mater. Interfaces 8 (2016) 2135821365

49) N.D. Nguyen, H.K. Kim, D.L. Nguyen, D.C. Nguyen and P.H.N Nguyen: Adv. Nat. Sci. Nanosci. Nanotechnol. 10 (2019) 015005.
50) G.T. Mola, X.G. Mbuyise, S.O. Oseni, W.M. Dlamini, P. Tonui, E.A.A. Arbab, K. Kaviyarasu and M. Maaza: Nano Hybrids Compos. 20 (2018) 90-107.

51) S. Pillai and M.A. Green: Sol. Energy Mater. Sol. Cells 94 (2010) 1481-1486.

52) J. Liu, H. He, D. Xiao, S. Yin, W. Ji, S. Jiang, D. Luo, B. Wang and Y. Liu: Materials (Basel) 11 (2018) 1833.

53) R. Ganesamoorthy, G. Sathiyan and P. Sakthivel: Sol. Energy Mater. Sol. Cells 161 (2017) 102-148.

54) H. Choi, J.P. Lee, S.J. Ko, J.W. Jung, H. Park, S. Yoo, O. Park, J.R. Jeong, S. Park and J.Y. Kim: Nano Lett. 13 (2013) 2204-2208.

55) J. Wang, S. Jia, Y. Cao, W. Wang and P. Yu: Nanoscale Res. Lett. 13 (2018) 211.

56) Q. Luo, C. Zhang, X. Deng, H. Zhu, Z. Li, Z. Wang, X. Chen and S Huang: ACS Appl. Mater. Interfaces 9 (2017) 34821-34832.

57) T.H. Hoang, T.G. Ho, V.H. Nguyen, T. Tran and V.T. Chu: Sens. Actuators B Chem. 249 (2017) 348-356.

58) R. Jain, A. Sinha, N. Kumari and A.L. Khan: Anal. Methods 8 (2016) 3034-3045.

59) I.B. Olenych, O.I. Aksimentyeva, L.S. Monastyrskii, Y.Y. Horbenko and L.I. Yarytska: Nanoscale Res. Lett. 10 (2015) 187.

60) I.B. Olenych, L.S. Monastyrskii, O.I. Aksimentyeva and B.S Sokolovskii: Ukr. J. Phys. 56 (2011) 1198-1202.

61) Y. Wang, L. Zhang, N. Hu, Y. Wang, Y. Zhang, Z. Zhou, Y. Liu, S. Shen and C. Peng: Nanoscale Res. Lett. 9 (2014) 251.

62) P. Patil, G. Gaikwad, D.R. Patil and J. Naik: J. Inst. Eng. Ser. D 97 (2016) 47-53.

63) K.J. Dunst, B. Scheibe, G. Nowaczyk, S. Jurga and P. Jasiński: Meas. Sci. Technol. 28 (2017) 054005.

64) P. Adelhelm, K. Cabrera and B.M. Smarsly: Sci. Technol. Adv. Mater. 13 (2012) 015010

65) B. Jache, C. Neumann, J. Becker, B.M. Smarsly and P. Adelhelm: J. Mater. Chem. 22 (2012) 10787-10794

66) Q.L. Nguyen, P.T. Tran, V.T. Duong, T.K. Pham, C.T. Nguyen, H.B. Luong, D.V. Dang, D.C. Nguyen and H.L. Nguyen: Mater. Trans. 56 (2015) 1399-1402.

67) M.V. Nguyen, N.T. Tran, T.H. Truong, D.C. Nguyen, D.Q. Nguyen, D Lee, D. Kim, T.L. Phan and D. Kim: Mater. Trans. 56 (2015) 1354 1357.

68) T. Tsutsui and S. Saito: Intrinsically Conduct. Polym. An Emerg. Technol., (Springer Netherlands, Dordrecht, 1993) pp. 123-134.

69) W. Tian, X. Liu and W. Yu: Appl. Sci. 8 (2018) 1118 (Review). 